\title{
Spontaneous isolated bladder rupture following normal delivery
}

\section{Valsa Diana*, Bhargavi K.}

Department of Obstetrics and Gynecology, RGGWCH, Puducherry, India

Received: 01 February 2018

Accepted: 07 March 2018

\section{*Correspondence:}

Dr. Valsa Diana,

E-mail: valsadiana@gmail.com

Copyright: (c) the author(s), publisher and licensee Medip Academy. This is an open-access article distributed under the terms of the Creative Commons Attribution Non-Commercial License, which permits unrestricted non-commercial use, distribution, and reproduction in any medium, provided the original work is properly cited.

\begin{abstract}
Spontaneous bladder rupture in a primipara after normal delivery within the first week of puerperium is a rare surgical emergency. The importance of suspecting and recognizing the voiding dysfunction in the postpartum period and early catheterisation to prevent such catastrophe is highlighted in this case report. Author report a case of spontaneous intraperitoneal bladder rupture in 26 years old primipara 3 days after normal delivery of a $3 \mathrm{~kg}$ baby with episiotomy.
\end{abstract}

Keywords: Bladder injury, Normal delivery

\section{INTRODUCTION}

Spontaneous bladder rupture during labour or puerperium of a normal vaginal delivery is an extremely rare condition. It may be associated with bladder diseases. The term spontaneous bladder rupture is applied to those cases in which there is neither a history of antecedent trauma nor any underlying bladder pathology with an Incidence of 1 in $126000 .^{1,2}$ Patient usually presents with acute abdomen complaining of supra pubic pain, anuria, haematuria. When it occurs, it is a surgical emergency and needs immediate diagnosis and treatment to decrease morbidity and mortality associated with this condition. Isolated intraperitoneal bladder rupture from a vaginal delivery is extremely occasional and the rupture of urinary bladder without a history of recent trauma or instrumentation is rare..$^{1,3,4}$

\section{CASE REPORT}

A 26-year-old primipara presented on PND 4 with retention of urine and abdominal distension for 2 days following a normal vaginal delivery. Labour was not prolonged and the delivery was uneventful with birth weight of $3 \mathrm{~kg}$. No history of trauma or previous abdominal surgery, history of radiation for any malignant disease, neurogenic bladder. She was catheterized, $1100 \mathrm{ml}$ of clear urine drained. Five days later she was again presented with complaints of pain abdomen, retention of urine and abdominal distension. Patient was febrile, abdominal distension with tenderness in the suprapubic region.

Lab reports showed leucocytosis and significant pyuria. Ultra sound results showed ascites with moving echoes, bilateral hydroureteronephrosis with cystitis, possibilities of peritonitis. Initial provisional diagnosis was possibilities of urosepsis or acute peritonitis. On the next day, the patient was deteriorated and developed tachypnoea and tachycardia, hypotension requiring monitoring in the intensive care unit with features of acute renal failure and septic shock.

CT Cystogram showed extravasation of contrast from bladder forming fistulas from dome of bladder to extraperitoneal area and a definitive diagnosis of isolated intraperitoneal bladder rupture was made. At exploratory laparotomy, abdomen was found to have purulent uricites with 4 rents of about $1-3 \mathrm{~cm}$ in dome and posterior wall of bladder. The bladder was repaired after debriding necroed tissue and drained via suprapubic and urethral catheter and an intraperitoneal drain in situ. A cystogram after 
4weeks showed no urinary leak and the suprapubic catheter was removed after 6 weeks. Postop period uneventful.

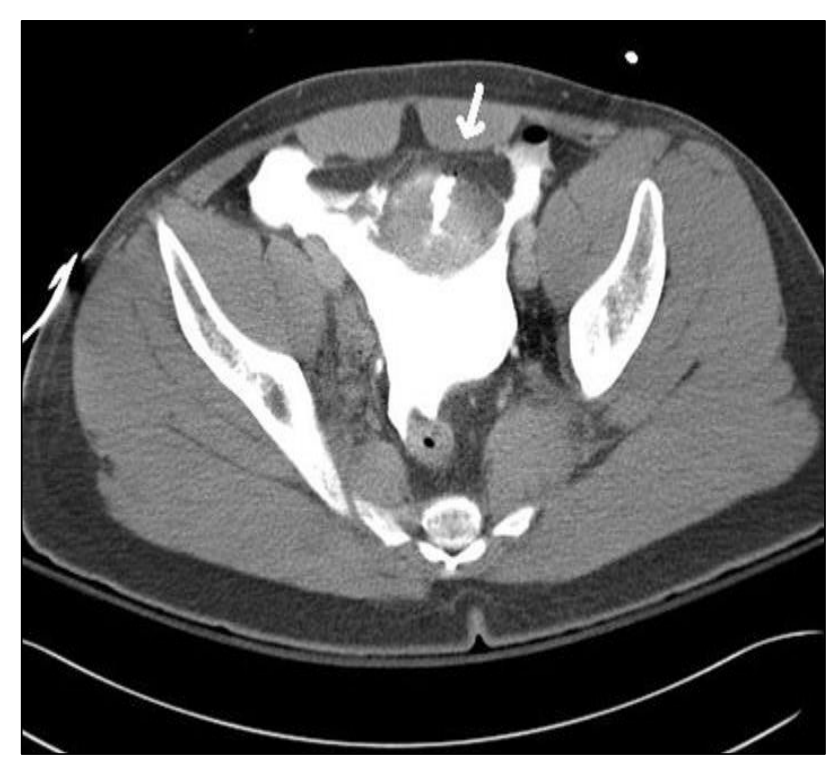

Figure 1: CT Cystogram showing intraperitoneal rupture.

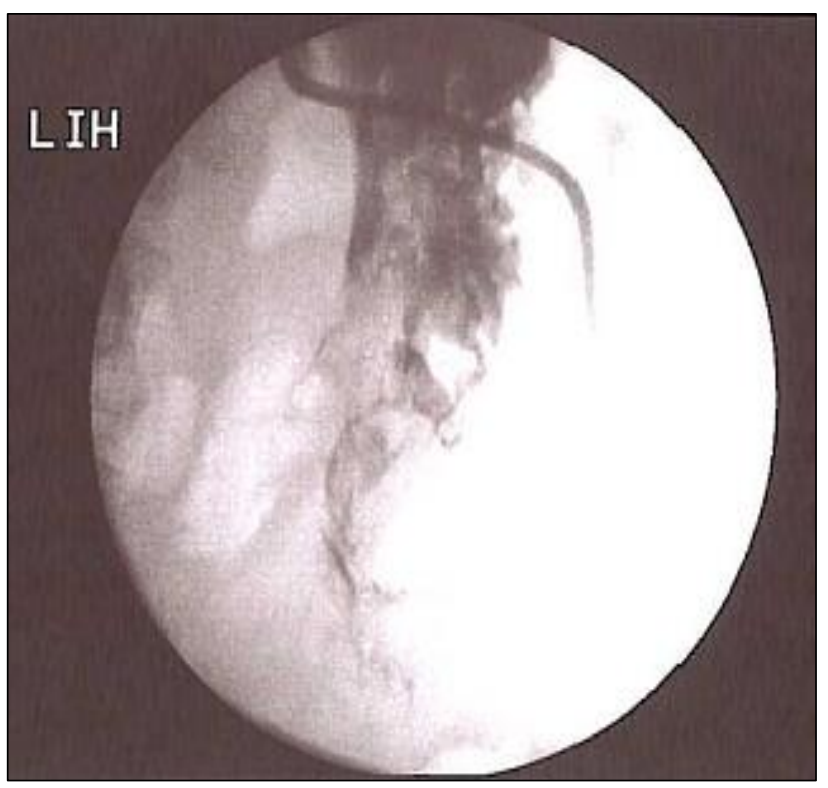

Figure 2: Contrast tracking down to peritoneum.

\section{DISCUSSION}

Bladder rupture in the puerperium is commonly associated with concomitant uterine rupture in the obstetric setting. Isolated intraperitoneal bladder rupture from a vaginal delivery is extremely rare. Kibel et al reported the first case in which the initial diagnosis was sepsis secondary to uterine rupture with renal failure on the basis of sepsis and NSAID use. ${ }^{1}$ Non-contrast CT was not useful for diagnosing the perforation. Diagnosis was made only during subsequent cystogram. The other case reported by Kekre et al was diagnosed as peritonitis with septicaemia. ${ }^{3}$ No cross-sectional imaging was done. Bladder perforation was detected only during laparotomy. Sandler et al, has described five types of bladder injuries with conventional cystography such as simple bladder contusion, intraperitoneal rupture, interstitial bladder injury, extraperitoneal bladder rupture and combination of intra and extraperitoneal bladder rupture. ${ }^{6}$ Any trauma to bladder can cause extraperitoneal and/or intraperitoneal ruptures.

Extra peritoneal rupture usually associated with pelvic fractures. Most common $80 \%$. Intraperitoneal bladder injury (20\%) usually follows penetrating trauma, following blunt trauma when bladder is overdistended and less commonly from iatrogenic causes. Intraperitoneal bladder rupture has been classified into two major groups depends on whether the rupture was due to a lesion of the bladder wall or caused by overdistension of the bladder from retention of urine. ${ }^{2}$ Retention of urine can result from three reasons such as Neurological disorders like tabs dorsalis, Urethral obstruction from impacted gravid uterus or fibroids and 'Miscellaneous' factors as puerperal and postoperative retention. The anatomic proximity of lower urinary tract and reproductive tract predisposes them to iatrogenic injury during Obstetric and Gynecological procedures. Commonly involved organs are bladder and ureter. ${ }^{7}$ The Trigone of bladder rests on anterior vaginal fornix and base rests on lower uterine segment and cervix. It is most frequently injured during obstetric procedures. ${ }^{8}$ Pathophysiology of the bladder rupture in puerperium includes sustained pressure from the fetal head against the bladder during forceful uterine contractions which may lead to pressure necrosis of the bladder dome. ${ }^{9}$ Other contributory factors include prolonged second stage and macrosomic babies. Bladder rupture during puerperium can occur either due to its incomplete evacuation from pain or from primary bladder pathology. Sudden onset of abdominal pain, generalized peritonitis and abnormal renal biochemistry leads to a high index of suspicion in such cases. But the pain in the second and third stages of labour is so severe that the pain due to rupture cannot be felt separately. So, patient may present late with features of renal failure and urinary ascites. In the postpartum patient with an uneventful labour presenting with ascites and azotaemia intraperitoneal rupture of the bladder should be the first suspect. A high index of clinical suspicion and retrograde cystography, CT cystogram, cystoscopy confirm the diagnosis. $1,3,4$

Early operative intervention with closure of the bladder rent and prolonged bladder drainage using both suprapubic and urethral catheter to minimize the urine leak reduces the morbidity. The most important thing to remember is that this condition is a totally preventable condition provided the bladder is empty during the second stage of the labour. So, emptying of the bladder should be done routinely in each patient before the patient proceeds to second stage of the disease. 


\section{CONCLUSION}

Postpartum patients who underwent episiotomy or perineal repair may land up in unnoticed urinary retention which may rarely terminate in spontaneous urinary bladder rupture. Awareness of the clinical, laboratory and radiology findings amongst emergency physician would help to initiate appropriate management in these patients.

Funding: No funding sources

Conflict of interest: None declared

Ethical approval: Not required

\section{REFERENCES}

1. Wandabwa J, Tom TO, Kiondo P. Spontaneous rupture of bladder in puerperium. Afr Health Sci. 2004;4:138-9.

2. Kekre AN, Kekre N, Nath V, Seshadri L. Spontaneous rupture of the urinary bladder in the puerperium. Aust NZJ Obstet Gynaec. 1997;37:4734.

3. Kibel AS, Staskin DR, Grigoriev VE. Intraperitoneal bladder rupture after normal vaginal delivery. J Urol. 1995;153:725-7.
4. Haddad FS, Wachtel TL. intraperitoneal rupture bladder. Urol Int. 1987;42:467-9.

5. Chan JK, Morrow J, Manetta A. Prevention of ureteral injuries during gynecological surgery. Am J Obstet Gynecol. 2003;188:1273.

6. Pandyan S, Zahrani AB, Awon AR, Rashid MA, Assiri MA, Dahanoun M. Iatrogenic bladder injuries during obstetric and gynecological procedures. Soudi Medical J. 2007;28(1):73-6.

7. Peters PC. Intraperitoneal rupture of the bladder. Urol Clin North Am. 1989;16:279-82.

8. Sandler CM, Hall JT, Rodriguez MB, Corriere JN Jr. Bladder injury in blunt pelvic trauma. Radiol. 1986;158:633-8.

9. Bastable JR, De Jode LR, Warren RP. Spontaneous rupture of the bladder. Br J Urol. 1959;31:78-86.

Cite this article as: Diana V, Bhargavi K. Spontaneous isolated bladder rupture following normal delivery. Int J Reprod Contracept Obstet Gynecol 2018;7:1638-40. 